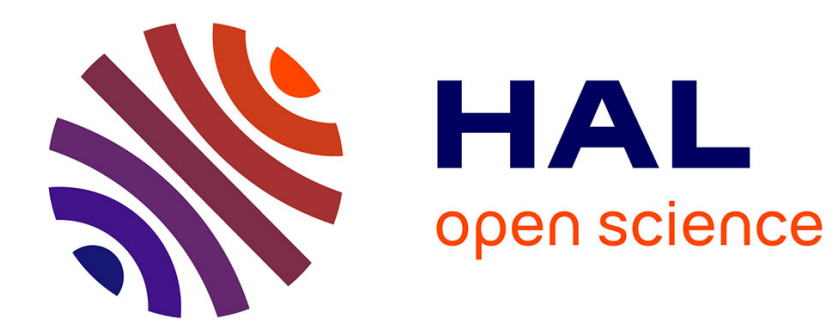

\title{
Sur la variation avec le temps de l'activité de quelques substances radioactives
}

\author{
Pierre Curie
}

\section{To cite this version:}

Pierre Curie. Sur la variation avec le temps de l'activité de quelques substances radioactives. Radium (Paris), 1911, 8 (10), pp.353-354. 10.1051/radium:01911008010035300 . jpa-00242489

\section{HAL Id: jpa-00242489 https://hal.science/jpa-00242489}

Submitted on 1 Jan 1911

HAL is a multi-disciplinary open access archive for the deposit and dissemination of scientific research documents, whether they are published or not. The documents may come from teaching and research institutions in France or abroad, or from public or private research centers.
L'archive ouverte pluridisciplinaire HAL, est destinée au dépôt et à la diffusion de documents scientifiques de niveau recherche, publiés ou non, émanant des établissements d'enseignement et de recherche français ou étrangers, des laboratoires publics ou privés. 


\title{
MÉMOIRES ORIGINAUX
}

\section{Sur la variation avec le temps \\ de l'activité de quelques substances radjoactives}

\author{
Par Mme P. CURJE
}

[Faculté des Sciences de Paris.]

D. puis plusicurs années des séries de mesures ont été organisées par moi dans mon laboratoire en vuc de suivre en fonction du temps l'activité des substances dont l'évolution est certainement très lente. Les substances qui ont été étudiées sont : l'uranium, l'actinium, le radioplomb ou radium D. Je me propose de communiquer ici les premiers résultats de ces séries de mesures.

Uranium. - L'étude de l'uranium est faite sur l'oxyde noir de ce corps. L'oxyde finement pulvérisé est distribué en couche uniforme sur la surface d'un plateau circulaire qui sert de plateau étalon. L'activité de ce plateau est mesurée par son rayonnement total dans une chambre d ionisation dans laquelle ce rayonnement est complètement absorbé, en ce qui concerne !es rayons $\alpha$. La mesure est faite par la méthode de compensation au moyen du quarı piezo-électrique. Aucune correction n'est faite pour tenir comple des variations de température el de pression qui n'ont pas d'influence sensible.

Les mesures précises ont été commencées en aoùt 1907. Elles s'étendent actuellement sur un intervalle de quatre années. Les différences entre les mesures individuelles, en général inférieures à 2 pour 100, peuvent exceptionnel'ement atteindre 5 à 4 pour 100; mais en comparant les mojennes des mesures elfectuées pendant chacune des quatre années on trouve que celles.ci ne diffèrent entre elles que de moins de 0,5 pour 100 . Un peut donc admettre que le rayonnement de l'uranium est resté constant pendant les quatre années écoulées à l'approximation indiquée.

Radium. - L'étude du radium esí faite par la mesure du rayonnement pénétrant de ce corps. Un sel de baryum radifère, contenant une petite proportion de sel de plomb, a été soumis à une purification, au mojen de la précipitation par l'hydrogène sulfuré. Celte opération a pour eflet de priver le sel des traces de radium D, E et $\mathbf{F}$ qui y sont contenues et qui se trouvent entrainées avec le plomb. L'émanation arant

T. 8. ensuite été chassée par ébullition de la solution, celle-ci a été rapidement évaporée; le sel séché a été enfermé dans une ampoule de verre longue et étroite, à parois minces; celte ampoule a ćté enveloppée d'une feuille d aluminium mince et utiliséc comme électrode centrale dans une chambre d'ionisation cylindrique. On mesure le courant d'ionisation dû au rayonnement pénétrant de l'ampoule, lequel est partiellement absorbé par l'air contenu dans la chambre d'onisation. Celle-ci n'étant pas hermétiquement close, la densité de l'air varie avec les conditions atmosphériques; la mesure du courant est donc sujette à une correction. On peut admettre, d'une manière approchée, que le courant varie proportionnellement ì la densité du gaz. Cette supposition, quoique certainement peu rigoureuse, permet de laire une correction qui rend les mesures bien plus régulières.

Les mesures poursuivies depuis vingt mois, mettent en évidence un accroissement lent de l'activilé de l'ampoule. Cet accroissement est au total de 4 pour 100 pour l'intervalle de temps considéré; il doit être d'environ 2,5 pour 100 par an pour les deux premières années.

Les rayons utilisés dans le cas actuel sont les rajons $\beta$ et les rayons $\gamma$, probablement surtout les premiers. On peut s'attendre à une augmentation du rayonnement par suite de la formation progressive de radium $\mathrm{E}$. Il est assez difficile de prévoir exactement la valeur de l'augmentation et des expériences spéciales sont nécessaires à cet effet. On peut cependant penser que l'augmentation observée est bien due à cette cause.

Actinium. - L'étude de l'actinium est faite de la même manière que celle du radium. Un sel d'actinium, qui arait été préparé par M. Debierne il y a plusieurs années, a été introduit dans une amponle de verre mince dont le rajonnement pénétrant est éludié de la même manière que celui de l'ampoule de radium. La même correction est apportée aux mesures. 
Les mesures se sont montries très peu régnlières, sans que l'on ait pu se rendre comple de la raison de ces irrégularilés. Cependant les expériences qui portent actuellement sur trois années, indiquent unc diminution assez importante de l'intensité du rayonnement. La diminution représente environ 10 pour 100 de la valeur du rayonnement primitif.

De quelle manière doit-on interpréter cette diminution? Il ne semble pas que le radioactinium ou l'actinium $X$ puissent intervenir, les mesures ayant été faites avec un sel qui arait atteint depuis longtemps son équilibre radioactif à ce point de vue. Nous pouvons donc supposer, ou bien qu'il existe entre l'actinium et le radioactinium unc substance intermediaire de longue vie moyenne, qui donne lieu à un rayonnement pénétrant et qui pouvait se trouver en excès dans le produit étudié, ou bien admettre que l'actinium n'est pas une substance de longue durée, ainsi qu on le crojait jusqu'à présent, mais qu'il se détruit avec une vitesse relativement grande. Les observations qui viennent d'être décrites conduiraient, en effet, à assigner à l'actinium une vie moyenne de l'ordre de 50 ans senlement. Par ce fait, la nécessité d'une relation entre l'actinium et les autres constituants radioactifs des minerais d'urane deviendrait tout à fait évidente. Cette question sera l'ohjet de recherches ultérieures.

Radium D. - Une expérience a été commencée en mai 1906 pour suivre l'évolution de l'activité du radioplomb, afin de la comparer à la théorie et afin de déterminer par l'expérience directe la période du radium $\mathrm{D}$.

Le plomb radioactif à l'état de chlorure a été soumis à une cristallisation dans l'eau additionnée d'acide chlorhydrique. Ce traitement a pour effet de séparcr le radium $\mathrm{E}$ et le polonium (radium $\mathrm{F}$ ) qui passent dans la dissolution. Le sel obtenu après plusieurs cristallisations n'a plus qu'un rayonnement $\alpha$ et un rayonnement $\beta$ insignifiants. L'évolution de chacun de ces rayonnements a été suivic pendant plusieurs années.

Le rayonnement pénétrant a atleint une valeur fixe en un mois. La loi de cette angmentation a conduit à assigner au radium $\mathbf{E}$ une période de 4,7 jours. Le rayonnement $\alpha$ a atteint son maximum en deux ans. Pendant la troisième annce le rayonnement $\%$ et le rayonnement $\beta$ se sont maintenus sensiblement conslants.

Si la période du radium D est supérieure à 15 ans, la loi exponentielle pour la décroissance du rajonnement $\alpha$ doit être établie quatre"années après la préparalion du sel inactif, à une approximation de 0,5 pour 100. Les expériences qui portent sur la cinquième amnée mettent en évidence une décroissance régulière de ce rayonnement; pendant $\mathbf{1} \mathbf{1 0}$ jours la diminution d'aclivité a été de 5 pour 100 de liı valeur observée au début de la cinquième année. Une conclusion précise est encore impossible, cependant on peut prévoir pour le radium D une période d'environ 17 ans et une vie morenne d'environ 20 ans. Les mesures faites sur le rayonnement $\beta$ ne sont pas assez précises pour permettre des conclusions sures; on peut dire seulement que ce rayonnement diminue, la vitesse de diminution étant du même ordre, mais plutôt plus faible que celle que l'on comptait observer.

Les séries de mesures dont les résultats viennent d'ètre indiqués, ont été effectuées avec le concours de M. Danysz et de M. Wertenstein que je remercie pour leur aide.

Toutes ces séries de mesures de longue durée sont effectuées par la méthode dı quartz piézo-électrique, servant comme étalon de quantité d'électricité. Quand la lame est bien montée, elle représente un ćtalon absolument constant. Quand il s'agit de mesures portant sur des intervalles de temps considírables, cette propriélé est particulièrement précieuse, car elle permet d'éviter les erreurs supplémentaires qui résultent du contrốle de la sensibilité de l'appareil de mesures utilisé. Ce contrôle peut ètre laissé de còté dans le cas actuel, ainsi que l'ont prouré les expériences d'étalonnage direct des lames de quartz, effectuées à des époques différentes avec une grande précision.

[Manuscrit rẹ̣ le 27 aoûl 1911.] 